\title{
Blood Harmane Concentrations in 497 Individuals Relative to Coffee, Cigarettes, and Food Consumption on the Morning of Testing
}

\author{
Elan D. Louis, ${ }^{1,2,3,4}$ Pam Factor-Litvak, ${ }^{3}$ Marina Gerbin,, ${ }^{1}$ Wendy Jiang, ${ }^{5}$ and Wei Zheng ${ }^{5}$ \\ ${ }^{1}$ GH Sergievsky Center, College of Physicians and Surgeons, Columbia University, Unit 198, Neurological Institute, \\ 710 West 168th Street, New York, NY 10032-2699, USA \\ ${ }^{2}$ Department of Neurology, College of Physicians and Surgeons, Columbia University, New York, NY 10027-6900, USA \\ ${ }^{3}$ Department of Epidemiology, Mailman School of Public Health, Columbia University, New York, NY 10032-3727, USA \\ ${ }^{4}$ Taub Institute for Research on Alzheimer's Disease and the Aging Brain, College of Physicians and Surgeons, \\ Columbia University, New York, NY 10032, USA \\ ${ }^{5}$ School of Health Sciences, Purdue University, West Lafayette, IN 47907-2051, USA
}

Correspondence should be addressed to Elan D. Louis, ed12@columbia.edu

Received 23 November 2010; Revised 11 February 2011; Accepted 16 February 2011

Academic Editor: Margaret James

Copyright ( 2011 Elan D. Louis et al. This is an open access article distributed under the Creative Commons Attribution License, which permits unrestricted use, distribution, and reproduction in any medium, provided the original work is properly cited.

\begin{abstract}
Harmane, a potent neurotoxin linked with several neurological disorders, is present in many foods, coffee, and cigarettes. We assessed whether morning food/coffee consumption and smoking were reflected in blood harmane concentrations (BHCs) we obtained in an epidemiologic sample $(n=497)$. Participants who smoked on the morning of phlebotomy had similar logBHCs to those who had not smoked $(P=.57)$; there was no correlation between logBHCs and number of cigarettes $(P=.59)$. Among the coffee drinkers, there was no correlation between number of cups and $\operatorname{logBHCs}(P=.98)$. Participants who had eaten on the morning of phlebotomy had similar $\log B \mathrm{HC}$ to those who had not $(P=.49)$; logBHCs did not correlate with the time latency between last food consumption and phlebotomy $(P=.74)$. BHCs in this sample of $\sim 500$ individuals did not covary with recent smoking, coffee, or food consumption, suggesting that our inability to withhold these exposures on the morning of phlebotomy was not reflected in the BHCs we measured.
\end{abstract}

\section{Introduction}

Harmane (1-methyl-9H-pyrido[3,4- $\beta$ ]indole) is a potent neurotoxin that has been linked with several neurological outcomes $[1,2]$. Although it is produced endogenously by the body, harmane is also present in many foods (esp. meats but also plant-derived foods) [3]. Studies have shown that harmane concentrations are particularly high in certain commonly consumed beverages (esp. coffee) [3-6] as well as cigarettes $[3,5,7]$. Coffee consumption and smoking are widespread and common human behaviors.

Smoking [8] and food ingestion [9] have been shown to result in transient elevations in BHCs. After smoking, blood harmane levels rise rapidly and seem to return to baseline within one hour, although the number of tested human volunteers has been small $(n=3)$ [8]. After oral dosing (harmane dissolved in corn oil), blood harmane levels in rats peaked rapidly (in approximately 30 minutes) and then gradually returned to baseline within 3-5 hours [9].

Studies of harmane and its relation to neurological outcomes (essential tremor [ET] and Parkinson's disease) often involve work with frail and elderly study subjects for whom fasting on the morning of testing is not feasible, especially as many of these patients must also take prescription medications (often accompanied with food). It is also difficult to ask smokers to refrain, and their attempts to do so can transiently exacerbate their tremor, confounding the accurate assessment of tremor severity. Given these limitations, it is important to know whether food consumption, coffee consumption, and/or smoking on the morning of phlebotomy are reflected in blood harmane concentrations (BHCs). 
Our overarching question was whether morning food/coffee consumption and smoking were reflected in the BHCs we obtained. The specific questions we asked were the following. (1) Did participants who smoked on the morning of phlebotomy have higher BHCs than those who did not smoke? (2) Was the the number of cigarettes smoked on the morning of phlebotomy correlated with BHCs? (3) Did participants who consumed coffee on the morning of phlebotomy have higher BHCs than those who did not? (4) Was the number of cups of coffee on the morning of phlebotomy correlated with BHCs? (5) Was there a correlation between the time of last food ingestion and BHCs? If these questions were answered affirmatively, this would suggest that these exposures are important to consider when assessing case-control differences in BHCs. If not, it would suggest that these exposures are relatively unimportant in this context. Using data from a large epidemiological study of ET, we evaluated these exposures in approximately 500 individuals. In addition to BHCs, information on smoking, coffee consumption, and food intake on the morning of phlebotomy were available.

\section{Methods}

2.1. Participants. All participants were enrolled between June 2000 and May 2008 in a study of the environmental epidemiology of tremor at Columbia-University Medical Center (CUMC). Participants consisted of ET cases and controls. By design, ET cases were identified from several sources; the major ones were a computerized billing database of patients at the Neurological Institute of New York, CUMC, and the International Essential Tremor Foundation, whose members were mailed advertisements $[2,10]$. All cases had received a diagnosis of ET from their treating neurologist and lived within two-hour driving distance of CUMC in the New York Metropolitan area. Based on a videotaped tremor examination, described below, their diagnoses were confirmed by a senior movement disorder neurologist (E.D.L.) using published diagnostic criteria (moderate or greater amplitude action tremor during $\geq 3$ activities or a head tremor in the absence of Parkinson's disease, dystonia, or another neurological disorder) [10-12].

Normal control subjects were also recruited during the same time period $[2,10]$. These controls were identified using random digit telephone dialing within a defined set of telephone area codes in the New York Metropolitan area that were represented by the ET cases. Controls were frequency matched to cases based on gender, race, and current age.

The CUMC Internal Review Board approved of all study procedures, and signed written informed consent was obtained from all participants upon enrollment $[2,10]$.

Of 698 ET cases and controls enrolled, complete data were available in 497 (71.2\%). The majority of the remaining participants had refused phlebotomy or had had an unsuccessful phlebotomy attempt. The final sample of 497 was similar to the base sample of 698 in terms of age (mean \pm standard deviation $=66.0 \pm 14.4$ versus $67.2 \pm 14.2$ years, $t=1.43$, and $P=.15$ ), gender (267 [53.7\%] versus 376
[53.9\%] female, chi-square $=0.00$, and $P=.96$ ), years of education $(15.4 \pm 3.5$ versus $15.3 \pm 3.5$ years, $t=0.49$, and $P=.63$ ), and proportion who were current smokers (43 $[8.7 \%]$ versus $57[8.2 \%])$ chi-square $=0.09$, and $P=.77)$.

2.2. Clinical Evaluation. All participants were evaluated in person by a trained tester. The tester administered clinical questionnaires and performed a videotaped tremor examination and phlebotomy.

As noted above, most evaluations were home visits and, therefore, were performed in the late morning, making fasting BHCs impractical.

The tester used a structured questionnaire to collect demographic information including age in years, gender, race, years of education, current smoker (yes versus no), the number of cigarettes smoked per day, cigarette pack years, the number of cigarettes smoked on the morning of phlebotomy, and the number of cups of coffee consumed on the morning of phlebotomy. Several years into the study, questions were added as to whether food was consumed on the morning of phlebotomy and the number of hours between last food consumption and the phlebotomy. Similar data on number of hours between smoking or coffee consumption and phlebotomy were not available. Medical comorbidity was assessed with the cumulative illness rating scale (range $=0$ 42 (high comorbidity)) [13].

The tester videotaped a tremor examination in all participants $[10,12]$. Each of 12 videotaped action tremor items was rated by a senior movement disorder neurologist (E.D.L.) on a scale from 0 (none) to 3 (severe tremor) [10-12].

2.3. BHCs. At the time of the evaluation, phlebotomy was performed. When the evaluation was performed in the participant's home, blood samples were temporarily stored on ice packs and then several hours later transferred to a $-20^{\circ} \mathrm{C}$ freezer; if performed at CUMC, they were placed immediately into a $-20^{\circ} \mathrm{C}$ freezer. Blood harmane concentrations were measured blinded to all clinical information with a well-established high-performance liquid chromatography method described in detail in our previous studies $[2,10,14]$. The intraday precision, measured as a coefficient of variation at $25 \mathrm{ng} / \mathrm{mL}$, was $6.7 \%$. The interday precision was $7.3 \%$ [14]. Our method uses whole blood rather than plasma. Harmane is highly lipophilic, accumulating inside of blood cells, with published studies demonstrating low relative recovery of harmane from plasma $[9,14]$.

2.4. Statistical Analyses. Statistical analyses were performed in SPSS (Version 18.0). The empirical distribution of BHCs was positively skewed (one-sample Kolmogorov-Smirnov test, $z=9.37, P<.001$ ), even after $\log$ transformation (one-sample Kolmogorov-Smirnov test, $z=2.37, P<.001$ ). Hence, nonparametric tests (Mann Whitney U, KruskalWallis, Spearman's rho) were used when assessing this variable. For smoking, pack years was assigned the value " 0 " for nonsmokers. We stratified participants based on tertiles of cigarettes smoked on the morning of phlebotomy. For 
TABLE 1: Characteristics of 497 study participants.

\begin{tabular}{lc}
\hline Age (years) & $66.0 \pm 14.4$ \\
Female gender & $267(53.7)$ \\
White race & $445(89.5)$ \\
Education (years) & $15.4 \pm 3.5$ \\
Cumulative illness rating scale score & $5.3 \pm 3.7$ \\
Current smoker & $43(8.7)$ \\
Number of cigarettes smoked per day (smokers only) & $18.8 \pm 17.0$ \\
Cigarette pack years & $10.8 \pm 20.9$ \\
Smoked on morning of phlebotomy & $43(8.7)$ \\
Number of cigarettes smoked on morning of & $0.4 \pm 1.5$ \\
phlebotomy & $214(43.1)$ \\
Drank coffee on morning of phlebotomy & $0.7 \pm 1.0$ \\
Number of cups of coffee on morning of phlebotomy & $0.45 \pm 0.60$ \\
\hline Log blood harmane concentration in ${ }^{-10} /$ ml & \\
\hline Values are means \pm standard deviation or number (percent). &
\end{tabular}

Values are means \pm standard deviation or number (percent).

coffee consumption, we dichotomized participants based on highest consumption level ( $\geq 4$ cups per day).

To assess potential confounding variables (e.g., education), stratified analyses were performed.

\section{Results}

3.1. Introduction. The 497 participants (242 ET cases and 255 controls) had a mean age of $66.0 \pm 14.4$ years (Table 1$)$. Of the $497,43(8.7 \%)$ had smoked on the morning of phlebotomy $(0.4 \pm 1.5$, range $=0-13$ cigarettes $)$, and $214(43.1 \%)$ had consumed coffee on the morning of phlebotomy $(0.7 \pm$ 1.0 , range $=0-5$ cups $)$. The mean log BHCs was $0.51 \pm$ $0.56 \mathrm{~g}^{-10} / \mathrm{mL}$ in ET cases and $0.38 \pm 0.63 \mathrm{~g}^{-10} / \mathrm{mL}$ in controls, a difference that was statistically significant (Mann Whitney $P=.01)$. The non-log-transformed BHCs was $11.49 \pm 52.05$ $($ median $=2.49) \mathrm{g}^{-10} / \mathrm{mL}$ in ET cases and $10.42 \pm 56.16$ (median $=1.81) \mathrm{g}^{-10} / \mathrm{mL}$ in controls. Log BHCs were not associated with age (Spearman's $r=0.02, P=.68$ ), gender (Mann Whitney $P=.80$ ), white race (Mann Whitney $P=$ .96), or cumulative illness rating scale score (Spearman's $r=-0.001, P=.99$ ), but they were weakly and inversely associated with years of education (Spearman's $r=-0.09$, $P=.04)$.

3.2. Smoking. Current smokers did not differ from current nonsmokers in terms of $\log$ BHCs $(0.39 \pm 0.52$ [median $=$ 0.20 ] versus $0.44 \pm 0.62$ [median $=0.34$ ], Mann Whitney $z=0.64, P=.52$ ). Additionally, neither cigarette pack years (Spearman's $r=-0.04, P=.42$ ) nor number of cigarettes smoked per day (Spearman's $r=0.04, P=.82$ ) were correlated with log BHCs.

Participants who smoked on the morning of phlebotomy had similar log BHCs to those who had not smoked on that morning $(0.41 \pm 0.51$ [median $=0.30$ ] versus $0.45 \pm 0.61$ [median $=0.34$ ], Mann Whitney $z=0.58$,
$P=.57)$. There was no correlation between $\log$ BHCs and the number of cigarettes smoked on the morning of phlebotomy (Spearman's $r=-0.03, P=.59$ ), even after restricting the analyses to the 43 participants who smoked on the morning of phlebotomy (Spearman's $r=0.10, P=.54$ ). High smokers (i.e., the 15 participants who were in the upper tertile of cigarettes smoked on the morning of phlebotomy, i.e., $\geq 5$ cigarettes) had similar log BHCs to the participants who had not smoked on the morning of phlebotomy $(0.51 \pm$ 0.54 [median $=0.20$ ] versus $0.45 \pm 0.61$ [median $=0.34$ ], Mann Whitney $z=0.20, P=.84$ ).

Years of education, which were associated with log BHCs, were inversely associated with number of cigarettes smoked on the morning of phlebotomy (Spearman's $r=-0.12$, $P=.008)$. Therefore, participants were stratified based on median years of education into a high ( $\geq 16$ years) versus low ( $<16$ years) education group; within these education strata, there was no difference in the log BHCs between participants who had versus who had not smoked on the morning of phlebotomy (for high education stratum, Mann Whitney $\mathrm{z}$ $=0.92, P=.36$; for low education stratum, Mann Whitney $z=0.05, P=.96)$. Participants were also stratified into those who had versus those who had not consumed coffee on the morning of phlebotomy; within these strata, there was no difference in the log BHCs between participants who had versus who had not smoked on the morning of phlebotomy (for coffee drinkers, Mann Whitney $z=0.31, P=.76$; for nondrinkers, Mann Whitney $z=0.90, P=.37)$. Finally, participants were stratified into cases versus controls; within these strata, there was no difference in the log BHCs between participants who had versus who had not smoked on the morning of phlebotomy (for cases, Mann Whitney $z=1.21$, $P=.23$; for controls, Mann Whitney $z=0.30, P=.76$ ).

3.3. Coffee. Participants who drank coffee on the morning of phlebotomy had a marginally lower log BHCs than those who had not had coffee on that morning $(0.39 \pm 0.59$ [median $=0.29$ ] versus $0.49 \pm 0.61$ [median $=0.38$ ], Mann Whitney $z=1.82, P=.069)$. ET cases had higher blood harmane than controls and, presumably to avoid exacerbating their tremor, also drank less coffee than controls. After stratifying by case-control status, drinking coffee on the morning of phlebotomy did not seem to be associated with higher log BHCs (Table 2).

In the combined sample of cases and controls, there was a marginal inverse correlation between log BHCs and the number of cups of coffee consumed on the morning of phlebotomy (Spearman's $r=-0.08, P=.08$ ) but not when analyses were restricted to the 214 participants who had consumed coffee on the morning of phlebotomy, Spearman's $r=0.002, P=.98$. High coffee consumers (i.e., the 11 participants who drank $\geq 4$ cups of coffee on the morning of phlebotomy) had marginally lower log BHCs than participants who had not had coffee on the morning of phlebotomy $(0.38 \pm 0.66$ [median $=0.29$ ] versus $0.49 \pm 0.61$ $[$ median $=0.38]$, Mann Whitney $z=1.72, P=.085)$. After stratifying by case-control status, there was a marginal, weak, inverse association between the number of cups of 
TABLE 2: Log blood harmane concentration and coffee consumption in analyses stratified by case-control status.

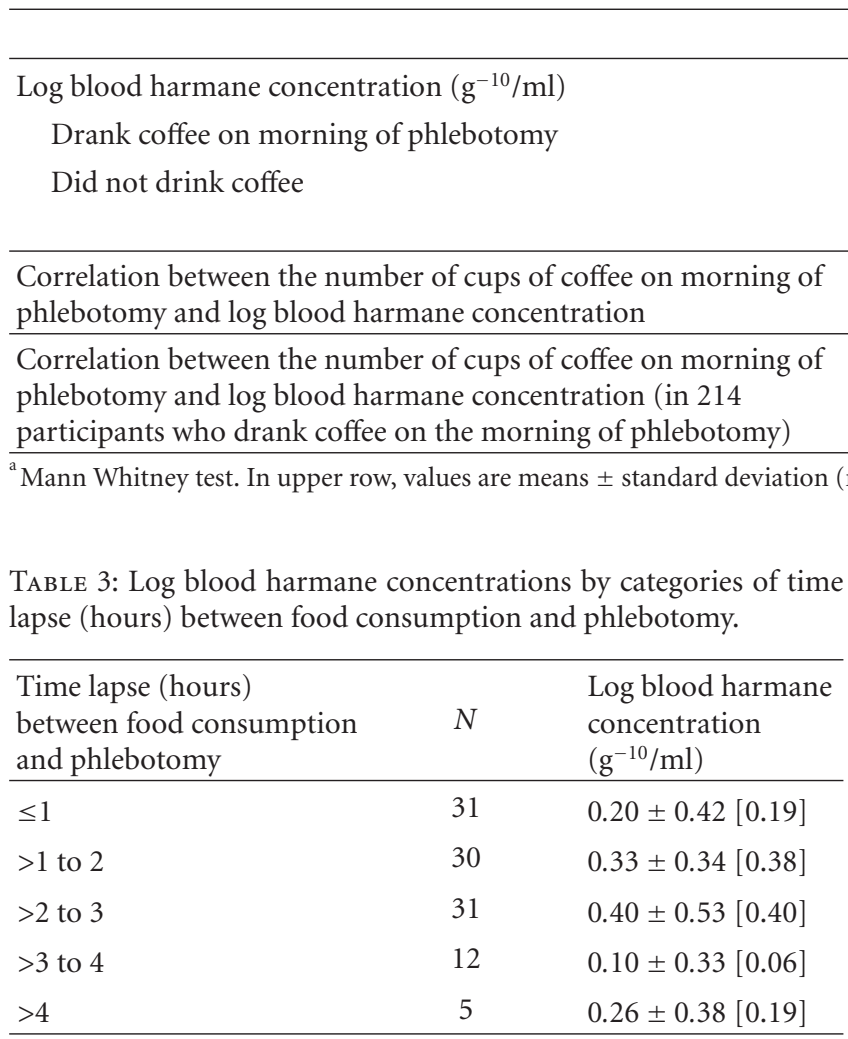

Values are means \pm standard deviation (median).

coffee and $\log$ BHCs in cases only but not when the analyses were restricted to the participants who had had coffee on the morning of phlebotomy (Table 2).

Years of education, which were associated with $\log$ BHCs, were not associated with the number of cups of coffee consumed on the morning of phlebotomy (Spearman's $r=$ $0.008, P=.86)$.

3.4. Food Consumption. Data were available on food consumption on the morning of phlebotomy in 215 participants, of whom $196(91.2 \%)$ had eaten on the morning of phlebotomy. The 196 participants who had eaten on the morning of phlebotomy had similar log BHCs to the 19 who had not eaten $(0.35 \pm 0.44$ [median $=0.33$ ] versus $0.34 \pm 0.47$ [median $=0.24$ ], Mann Whitney $z=0.70$, and $P=.49)$. After stratifying by case-control status, the results were similar in cases: $(0.42 \pm 0.47$ [median $=0.38$ ] in 115 eaters versus $0.37 \pm 0.47$ [median $=0.24$ ] in 7 noneaters, Mann Whitney $z=0.48$, and $P=.63$ ) and in controls $(0.25 \pm 0.39$ [median $=0.26]$ in 81 eaters versus $0.32 \pm 0.48$ [median $=0.24$ ] in 12 noneaters, Mann Whitney $z=0.18$, and $P=.86$ ).

Data were available for 109 participants on the time lapse between last food consumption and phlebotomy. The mean lapse was $2.0 \pm 1.1$ hours [median $=1.8$ ], range $=0-5.5$ hours. There was no correlation between the duration of this time lapse and log BHCs (Spearman's $r=0.03, P=$ .74). After stratifying by case-control status, the results were
ET cases $(N=242)$ Controls $(N=255)$

$$
\begin{gathered}
0.42 \pm 0.54[0.34] \\
0.56 \pm 0.57[0.42] \\
P=.11^{\mathrm{a}}
\end{gathered}
$$$$
0.37 \pm 0.62[0.28]
$$$$
0.40 \pm 0.65[0.26]
$$$$
P=.69^{\mathrm{a}}
$$

$r=-0.012, P=.068$

$r=-0.002, P=.97$

$r=-0.014, P=.23$

$r=0.078, P=.38$

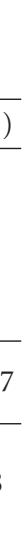


question whether food consumption, coffee consumption, and/or smoking, which often occur on the morning of phlebotomy, influence BHCs. The current dataset allowed us to examine this set of questions in a sample of approximately 500 study participants with both exposure data on these lifestyle characteristics and data on BHCs.

We found that participants who had smoked on the morning of phlebotomy had similar BHCs to their counterparts who had not smoked that morning. Furthermore, there was no detectable association between dosage of exposure to cigarettes on the morning of phlebotomy and BHCs. A cautionary note is that we did not collect blood samples within one hour of smoking, and, as discussed in detail below, this could have accounted for the lack of association between smoking and BHCs. For coffee consumption, there was only a very marginal inverse relationship with BHCs, but this was not consistent and, hence, of questionable significance. Finally, in the subsample of participants with timed data on food consumption, there was no discernable correlation between the time of the last food ingestion and BHCs. These results were observed in parallel in normal controls as well as in a group of ET patients.

How do our results relate to those from prior studies? After smoking, blood harmane levels rise rapidly and seem to return to baseline within one hour [8], which suggests that the rise is transient and time limited. In another study of 39 human volunteers, plasma concentrations of norharman (9H-pyrido[3,4- $\beta]$ indole), a neurotoxin that is structurally very similar to harmane, were on average several fold higher when smokers were not restricted in terms of smoking as compared to a condition in which the same smokers had refrained from smoking for at least six hours [15]. Another study [16], of 20 human volunteers, found a positive correlation between number of cigarettes smoked per day and plasma harmane concentration. It is possible that participants in these two prior studies smoked with one hour of phlebotomy, thereby resulting in acute elevations in BHCs and a detectable correlation between smoking behavior and BHCs. We did not collect data on the time that our participants smoked, so we do not know how many smokers smoked within one hour of phlebotomy. If most did not, this could explain our null finding.

In rats, oral dosing of harmane (i.e., $110 \mathrm{umol} / \mathrm{kg}$ harmane dissolved in corn oil) caused blood harmane levels to peak rapidly (in approximately 30 minutes) and then gradually return to baseline within 3-5 hours [9]. Yet, a study involving humans showed a range of responses to ingestion of ethanol or orange juice, including transient declines and, in others, transient rises in BHCs, with these transient changes ranging in duration from 1 hour to more than 8 hours [17]. Hence, the effects of food ingestion on BHCs in humans are less clear than the effects of oral dosing of harmane in laboratory animals. Our current results did not find any association between food intake or time of food intake and BHCs.

This study had limitations. This was not a pharmacokinetic study, and we did not assess pre- versus postconsumption BHCs in order to directly test whether blood concentrations rose. Furthermore, while in a subsample of participants, we had data on the time latency between the last food ingestion and phlebotomy, we had no data on the time of the last cigarette use or coffee consumption and phlebotomy, so for cigarettes and coffee, we were unable to time lock the events to phlebotomy. Second, we limited our assessment of smoking to cigarettes (i.e., inhaled smoke products) rather than pipe smoke or chewing tobacco, which were rare. Third, we did not assess whether different brands of coffee or cigarettes or differences in cooking styles influenced the BHCs we observed. The study also had several strengths. First, both BHCs and clinical data were assessed in the same study sample, comprising a collection of nearly 500 individuals. Second, for smoking, we presented both historical data for the degree of their regular consumption as well as data on acute consumption on the morning of phlebotomy. Third, the results were observed in normal controls and then reproduced in ET patients. Fourth, most published studies determined harmane concentrations in plasma, whereas our study investigates harmane in whole blood. The latter approach allows for a more accurate assessment of harmane concentrations by including all blood compartments. Finally, there is the uniqueness of the question; there are no other studies that have examined this issue.

\section{Conclusions}

In summary, BHCs in this sample of nearly 500 individuals did not covary with smoking, coffee consumption, or food consumption, suggesting that our inability to withhold these exposures on the morning of phlebotomy was not reflected in the BHCs we measured.

\section{Financial Disclosure}

E. D. Louis was funded by R01 NS39422, P30 ES09089, and RR00645 (General Clinical Research Center) from the National Institutes of Health (Bethesda, MD and Research Triangle, NC). P. Factor-Litvak was funded by R01 ES12231 and R01 ES017024 from the National Institutes of Health (Research Triangle, NC). W. Zheng was funded by R01 NS39422, R01 ES008146, and R21 ES017055 from the National Institutes of Health (Research Triangle, NC). The National Institutes of Health played no role in the study design, the collection of data, the analysis and interpretation of data, the writing of the paper, or in the decision to submit the paper for publication. The authors were free to design, conduct, interpret, and publish research, and this was not compromised by the National Institutes of Health.

\section{Conflict of Interests}

The authors declare that there is no conflict of interests. The statistical analyses were conducted by E. D. Louis.
Abbreviations
BHCs: Blood harmane concentrations
CUMC: Columbia-University Medical Center
ET: $\quad$ Essential tremor. 


\section{References}

[1] W. Kuhn, T. Muller, H. Grosse, and H. Rommelspacher, "Plasma harman and norharman in Parkinson's disease," Journal of Neural Transmission, Supplement, no. 46, pp. 291295, 1995.

[2] E. D. Louis, W. Jiang, K. M. Pellegrino et al., "Elevated blood harmane (1-methyl-9H-pyrido[3,4-b]indole) concentrations in essential tremor," NeuroToxicology, vol. 29, no. 2, pp. 294300, 2008.

[3] W. Pfau and K. Skog, "Exposure to $\beta$-carbolines norharman and harman," Journal of Chromatography B, vol. 802, no. 1, pp. 115-126, 2004.

[4] T. Herraiz, "Identification and occurence of the bioactive $\beta$ carbolines norharman and harman in coffee brews," Food Additives and Contaminants, vol. 19, no. 8, pp. 748-754, 2002.

[5] T. Herraiz, "Relative exposure to $\beta$-carbolines norharman and harman from foods and tobacco smoke," Food Additives and Contaminants, vol. 21, no. 11, pp. 1041-1050, 2004.

[6] T. Herraiz and C. Chaparro, "Human monoamine oxidase enzyme inhibition by coffee and $\beta$-carbolines norharman and harman isolated from coffee," Life Sciences, vol. 78, no. 8, pp. 795-802, 2006.

[7] Y. Totsuka, H. Ushiyama, J. Ishihara et al., "Quantification of the co-mutagenic $\beta$-carbolines, norharman and harman, in cigarette smoke condensates and cooked foods," Cancer Letters, vol. 143, no. 2, pp. 139-143, 1999.

[8] U. Breyer-Pfaff, G. Wiatr, I. Stevens, H. J. Gaertner, G. Mundle, and K. Mann, "Elevated norharman plasma levels in alcoholic patients and controls resulting from tobacco smoking," Life Sciences, vol. 58, no. 17, pp. 1425-1432, 1996.

[9] Y. Guan, E. D. Louis, and W. Zheng, "Toxicokinetics of tremorogenic natural products, harmane and harmine, in male sprague-dawley rats," Journal of Toxicology and Environmental Health Part A, vol. 64, no. 8, pp. 645-660, 2001.

[10] E. D. Louis, W. Zheng, E. C. Jurewicz et al., "Elevation of blood $\beta$-carboline alkaloids in essential tremor," Neurology, vol. 59, no. 12, pp. 1940-1944, 2002.

[11] E. D. Louis, B. Ford, H. Lee, and H. Andrews, "Does a screening questionnaire for essential tremor agree with the physician's examination?" Neurology, vol. 50, no. 5, pp. 13511357, 1998.

[12] E. D. Louis, R. Ottman, B. Ford et al., "The Washington Heights-Inwood Genetic Study of Essential Tremor: methodologic issues in essential-tremor research," Neuroepidemiology, vol. 16, no. 3, pp. 124-133, 1997.

[13] B. S. Linn, M. W. Linn, and L. Gurel, "Cumulative illness rating scale," Journal of the American Geriatrics Society, vol. 16, no. 5, pp. 622-626, 1968.

[14] W. Zheng, S. Wang, L. F. Barnes, Y. Guan, and E. D. Louis, "Determination of harmane and harmine in human blood using reversed-phased high-performance liquid chromatography and fluorescence detection," Analytical Biochemistry, vol. 279, no. 2, pp. 125-129, 2000.

[15] R. Spijkerman, R. van den Eijnden, D. van de Mheen, I. Bongers, and D. Fekkes, "The impact of smoking and drinking on plasma levels of norharman," European Neuropsychopharmacology, vol. 12, no. 1, pp. 61-71, 2002.

[16] F. Kiefer, A. Krödel, H. Jahn, K. Wolf, and A. Barocka, "Harman and norharman plasma levels in weaned alcoholics: correlations with depression and tobacco smoking," Addiction Biology, vol. 5, no. 4, pp. 437-441, 2000.
[17] H. Rommelspacher, L. G. Schmidt, and T. May, "Plasma norharman ( $\beta$-carboline) levels are elevated in chronic alcoholics," Alcoholism: Clinical and Experimental Research, vol. 15, no. 3, pp. 553-559, 1991. 

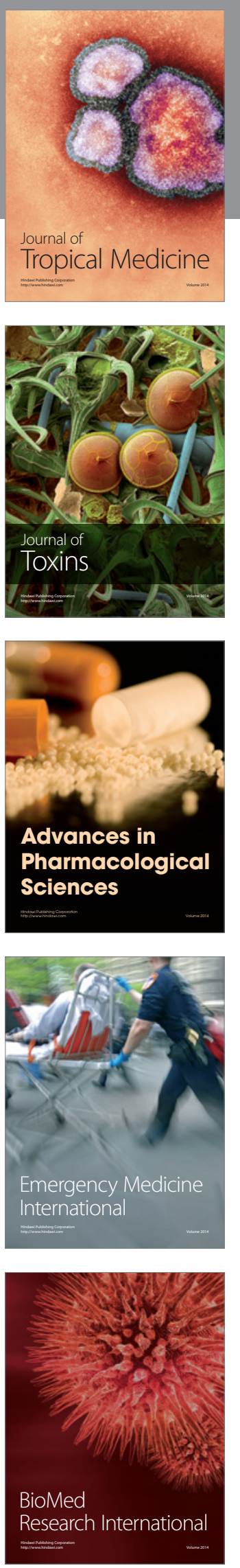
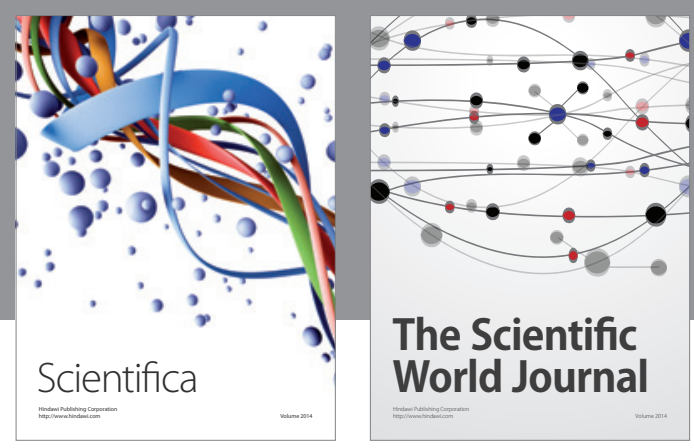

The Scientific World Journal
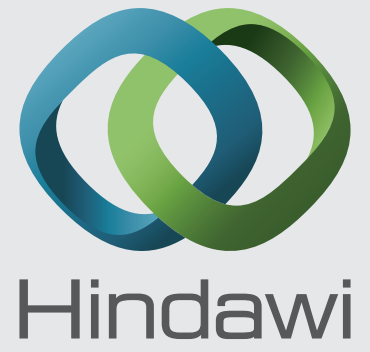

Submit your manuscripts at

http://www.hindawi.com
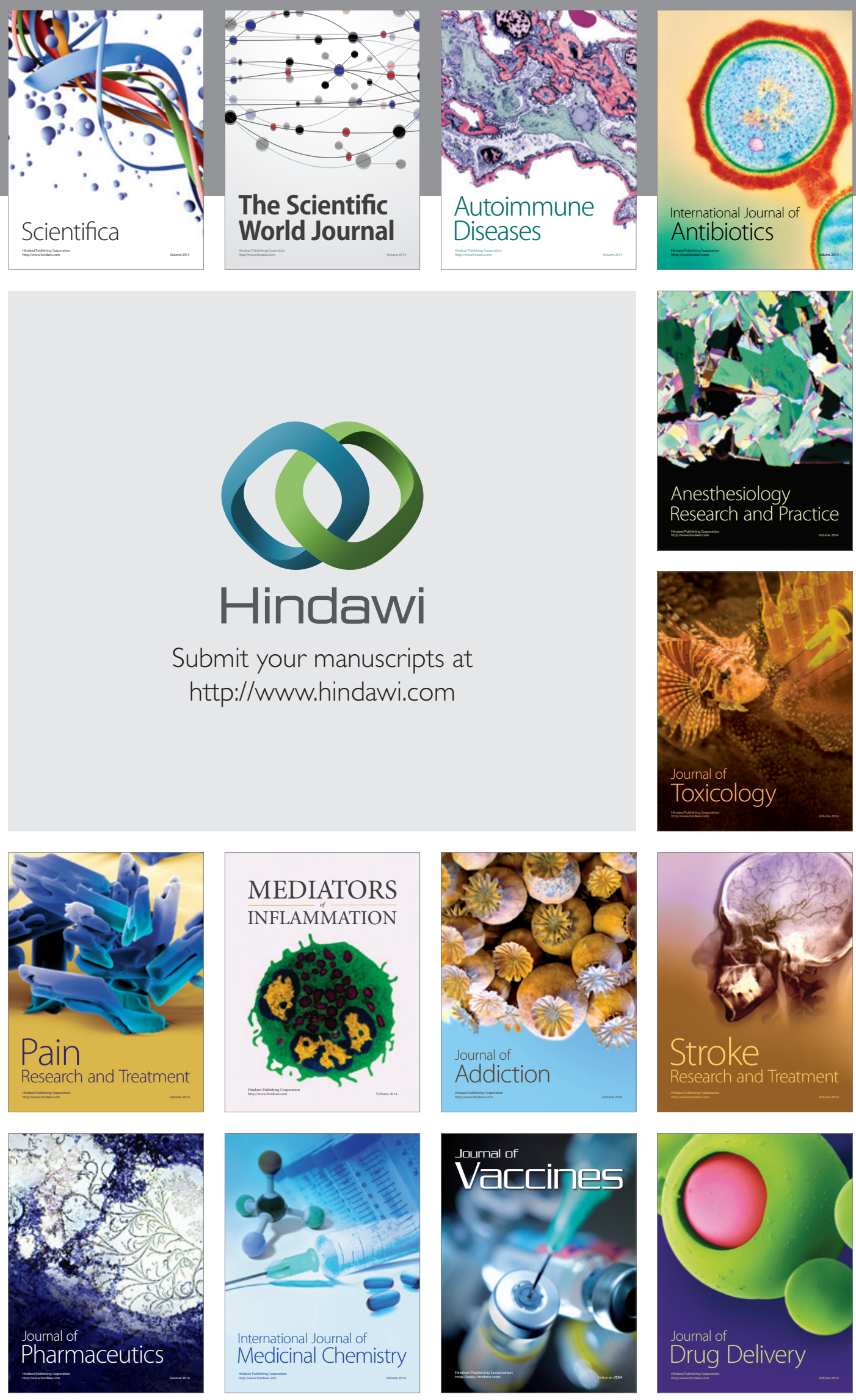